Juan P. Padilla-Martinez, Darren Banks, Julio C. Ramirez-San-Juan, Ruben Ramos-Garcia, Feng Sun and Guillermo Aguilar*

\title{
Towards the enhancement of transdermal drug delivery through thermocavitation
}

\author{
Verbesserung der transdermalen Wirkstoffverabreichung durch Thermokavitation
}

\section{Abstract}

Background and objective: Although for some highly lipophillic drugs the principal barrier to permeate the human skin may reside in the essentially viable epidermal membrane, for most molecules, the stratum corneum (SC) is the rate-limiting barrier to drug delivery. Today, several techniques have been developed to enhance transdermal drug delivery (TDD) by increasing the effective permeability of the SC (e.g., iontophoresis, electroporation, micro-needle, ultrasound, radio frequency and laser radiation). The goal of this study is to investigate the extent to which thermocavitation may be used as a novel alternative method to selectively pierce the SC and thus enhance TDD. Thermocavitation for this purpose is generated by a continuous wave (CW), low power laser beam focused on a highly-absorbing solution topically applied on the skin surface. The absorbed light creates a superheated volume in a tightly localized region followed by explosive phase transition and the formation of vapor-gas bubbles, which expand and later collapse very rapidly emitting intense acoustic shockwaves that disrupt the surface underneath. Materials and methods: Thermocavitation bubbles were induced close to the surface of skin models (agar gels) and ex-vivo porcine skin samples using a $975 \mathrm{~nm}$ CW laser, focused on a thin (100-300 $\mu \mathrm{m})$ topical layer of copper nitrate $\left(\mathrm{CuNO}_{4}\right)$. The damage induced by thermocavitation on the surface of agar tissue phantoms was analyzed by optical microscopy and the penetration depth of a fluorescent drug surrogate (FITC dextran, molecular weight $=4$ $\mathrm{kDa}$ ), applied topically to the surface of ex-vivo porcine skin samples following thermocavitation. The corresponding histological structure was analyzed by fluorescent microscopy and hematoxylin and eosin (H\&E) staining, respectively.

Results: The damage observed on agar gel and porcine skin appears to be congruent with the relationship between laser power, focal point, cavitation frequency and extent of damage observed in previous studies. In particular, the greatest damage induced to the agar phantoms was produced with the lowest laser power $(\sim 153 \mathrm{~mW})$ and thinnest solution layer $(\sim 100 \mu \mathrm{m})$ used. Similar laser and solution layer settings led to porcine skin damage of $\sim 80-100 \mu \mathrm{m}$ in diameter, which was sufficiently large to break the SC and allow the penetration of $4 \mathrm{kDa}$, FITCdextran to depths of $\sim 40-60 \mu \mathrm{m}$.

Conclusion: This novel approach to achieve cavitation is attractive and seems promising because it can be generated with inexpensive, low power CW lasers, capable of selectively disrupting the $\mathrm{SC}$ and allowing the penetration of large, hydrophilic drugs topically applied to the skin.

Keywords: thermocavitation; stratum corneum; tape stripping; H\&E technique; drug delivery.

\section{Zusammenfassung}

Hintergrund und Zielsetzung: Das Eindringen einiger lipophiler Wirkstoffe in die menschliche Haut wird durch die epidermale Membran begrenzt. Für die meisten Moleküle stellt aber das Stratum corneum (SC) die Barriere für die Wirkstoffverabreichung dar. Es sind verschiedene Techniken zur Wirkstoffverabreichung entwickelt worden, um die effektive Permeabilität des SC zu erhöhen (z.B. durch Iontophorese, Elektroporation, Mikronadeln, Ultraschall, Hochfrequenz, Laserstrahlung). Ziel der vorliegenden Studie war es zu untersuchen, ob das SC mittels Thermokavitation gezielt perforiert werden kann, um so die Durchlässigkeit für die Wirkstoffverabreichung zu verbessern. Thermokavitation kann erreicht werden, indem der Strahl eines kontinuierlich abstrahlenden (continuous wave, cw) Low-Power-Lasers auf die Hautoberfläche fokussiert wird, auf die zuvor eine stark absorbierende Lösung topisch aufgetragen wurde. Das absorbierte Licht erzeugt in einem begrenzten Volumenbereich eine Überhitzung gefolgt von einem explosiven Phasenübergang und der Formierung von Dampfgasblasen, die 
expandieren und schließlich extrem schnell kollabieren. Die dabei erzeugten akustischen Schockwellen zerreißen die Gewebeoberfläche.

Material und Methoden: Thermokavitationsblasen wurden dicht an der Oberfläche von Hautphantomen (Agargel) und Ex-Vivo-Hautproben (Schwein) mittels eines $975 \mathrm{~nm}$, cw Lasers erzeugt, indem der Laserstrahl auf dünne Schichten $(100-300 \mu \mathrm{m})$ von Kupfernitrat $\left(\mathrm{CuNO}_{4}\right)$ fokussiert wurde. Die dadurch erzeugten oberflächlichen Schädigungen wurden bei den Agarphantomen mittels optischer Mikroskopie evaluiert. Von den Ex-Vivo-Hautproben wurden zusätzlich HE-Schnitte angefertigt und bewertet sowie die Eindringtiefe eines fluoreszierenden Wirkstoffsurrogates (FITC-Dextran, Molekulargewicht $=4$ kDa) mittels Fluoreszenzmikroskopie bestimmt.

Ergebnisse: Die beobachteten Schädigungen an Agargel und Schweinehaut stimmen mit früheren Studien überein und spiegeln den Zusammenhang zwischen Laserleistung, Fokus, Kavitationsfrequenz und Schädigungsausmaß. Die größte Schädigung in Agargel wurde mit der geringsten Laserleistung ( $\sim 153 \mathrm{~mW})$ und der dünnsten Schichtdicke $(\sim 100 \mu \mathrm{m})$ erzeugt. Mit den gleichen Parametern konnten bei der Schweinehaut Schäden mit einem Durchmesser von ca. 80-100 $\mu \mathrm{m}$ erzeugt werden, was ausreichend war um das SC aufzubrechen, so dass das FITC-Dextran ca. 40-60 $\mu \mathrm{m}$ tief eindringen konnte.

Schlussfolgerung: Das vorgestellte Verfahren zur Erzeugung von Thermokavitation erscheint vielversprechend, zumal preiswerte cw Low-Power-Laser eingesetzt werden können. Es ist geeignet, um das SC selektiv zu perforieren, so dass große, hydrophile Wirkstoffe, die zuvor topisch auf die Haut aufgetragen wurden, eindringen können.

Schlüsselwörter: Thermokavitation; Stratum corneum; Tapestripping; H\&E-Färbetechnik; Wirkstoffverabreichung.

\footnotetext{
*Corresponding author: Guillermo Aguilar, Department of Mechanical Engineering, University of California Riverside, A-345 Bourns Hall, Riverside CA, 92521, USA, e-mail: gaguilar@engr.ucr.edu Juan P. Padilla-Martinez: Instituto Nacional de Astrofísica, Óptica y Electrónica, Departamento de Óptica, Apartado Postal 51 y 216, Puebla, Puebla 72000, México

Darren Banks: Department of Mechanical Engineering, University of California Riverside, A-345 Bourns Hall, Riverside CA, 92521, USA

Julio C. Ramirez-San-Juan: Instituto Nacional de Astrofísica, Óptica y Electrónica, Departamento de Óptica, Apartado Postal 51 y 216, Puebla, Puebla 72000, México

Ruben Ramos-Garcia: Instituto Nacional de Astrofísica, Óptica y Electrónica, Departamento de Óptica, Apartado Postal 51 y 216, Puebla, Puebla 72000, México

Feng Sun: Department of Mechanical Engineering, University of California Riverside, A-345 Bourns Hall, Riverside CA, 92521, USA
}

\section{Introduction}

Cavitation is a well-known phenomenon that has found applications in many research fields, including biomedicine. By far the most common cavitation applications are produced through the use of ultrasound generated by transducers (acoustic cavitation). This approach, referred to as lithotripsy, is well known due to its ability to disintegrate kidney and gall stones so that they can be rejected through the urinary tract [1-3]. Acoustic cavitation is also a well sought approach for other forms of controlled tissue damage including thrombolysis [4], lipoplasty [5], wound healing [6], fracture healing [7], and sonoporation [8-11]. Another method of generating cavitation in a selected region is by means of a focused, pulsed laser beam, known as photodisruption. This approach creates cavitation bubbles that cut through tissue and, thus, generate precise microscopic incisions of great value in many surgical procedures [12, 13]. Acoustic cavitation plays a particularly important role in enhancing transdermal transport of macromolecules, thereby offering a non-invasive mode of drug delivery (sonophoresis), where the collapse of cavitation bubbles microscopically disrupt the lipid bilayers of the stratum corneum (SC) to allow the permeation of drugs [14-17].

In the present paper, the potential of the relatively unexplored method of thermocavitation, as advanced by Rastopov and Sukhodolsky $[18,19]$ and recently reviewed by Ramirez-San-Juan et al. [20], i.e., cavitation induced by continuous wave (CW) low power lasers in absorbing solutions, is explored as an alternative method to enhance the permeation of the SC. In previous work it was shown that the shock waves generated immediately after the collapse of thermocavitation bubbles [21] are energetic enough to produce damage in materials as hard as titanium and indium tin oxide thin films [22]. Therefore, in this work the damage induced on softer materials, such as agar gels and ex-vivo porcine skin itself is studied as a preamble to studies on thermocavitation for tissue ablation, which may result in the enhancement of transdermal drug delivery (TDD), including that of optical clearing agents [23].

\section{Materials and methods}

\subsection{Sample preparation}

Two types of samples were used for these experiments: (a) tissue phantoms made of agar gel layers (BD Bioscience, Franklin Lakes, NJ, USA) prepared with $1 \%$ to $6 \%$ agar concentrations and (b) ex-vivo porcine skin obtained 
from Sierra for Medical Science (Whittier, CA, USA). On the upper surface of both sample types, a thin layer of saturated copper nitrate solution $\left(13.78 \mathrm{~g}\right.$ of $\mathrm{CuNO}_{4}$ per $10 \mathrm{ml}$ of water) was applied and a cover slip was placed on top (see Figure 1A). Using pre-manufactured plastic spacers, it was ensured that the thickness of the solution layer "sandwiched" between the cover slip and the sample surface was set to 100, 200 or $300 \mu \mathrm{m}$. The agar samples were prepared by weight percentage (e.g., for $2 \%$ concentration, $2 \mathrm{~g}$ of agar powder were dissolved in 100 $\mathrm{ml}$ of deionized water). For each sample, the initial water content was split in two equal parts. The agar was added to one half as the other half was boiled. Once the second portion was boiling, the first portion with dissolved agar was added, and the sample was heated until air bubbles stopped forming within the mixture. The sample was then poured into its final container that ensured $7 \mathrm{~mm}$-thick layers once the solution solidified (gelled). The porcine skin samples of $2 \mathrm{~cm}^{2}$ were carefully processed in the lab. Hairs were trimmed off using tissue scissors close to the skin surface but making sure the skin surface remained intact to avoid rupture of the SC and thus unintended alteration of the skin permeability. Finally, subcutaneous fat and muscle tissue were removed, and the skin sample surface was washed using deionized water. Thereafter, skin samples were snap-frozen in liquid nitrogen $\left(\mathrm{LN}_{2}\right)$ and stored in the freezer until thawed in phosphate buffer saline (PBS) for $1 \mathrm{~h}$ before experiments.

\subsection{Thermocavitation experiments}

A CW fiber laser (Mod. BWF1; B\&W TEK Inc., Newark, USA) with wavelength of $\lambda=975 \mathrm{~nm}$ was collimated and focused on the bottom surface of a cover slip in contact with the solution layer using a microscope objective ( $\mathrm{f}=8$ $\mathrm{mm}$ ), which produced a spot of $\sim 2 \mu \mathrm{m}$ in diameter at the focal point (Figure 1A). In order to observe the position of the laser beam's waist, a dichroic mirror was used to redirect the laser light reflected by the glass-liquid interface onto a CCD camera (Figure 1A). Since the Rayleigh distance of the lens is $\sim 13 \mu \mathrm{m}$, this reflection is a good indicator of the beam's waist diameter on the cover slip, although its exact location is not too critical for our measurements [24]. The absorption coefficient of the saturated solution at this wavelength, $\alpha=135 \mathrm{~cm}^{-1}$ [20], is so large that it warranties minimal light radiation on the skin and, therefore, minimal risk of thermal damage, so all experiments were performed with this solution. Light absorbed near the cover slip's bottom surface, heats the solution up to its critical limit, i.e., the temperature $\left(-270-300^{\circ} \mathrm{C}\right)$ at which an explosive liquid-gas phase transition occurs [2527]. This leads to the generation of multiple vapor bubbles that grow and collapse very rapidly emitting intense acoustic shock waves [18-21]. In CW thermocavitation, the maximum radius of the bubble can be controlled through the beam power or the beam's waist position [21]. To simplify the experiments, the beam's waist position was fixed on the cover slip's bottom surface and only the laser power and/or the thickness of the solution layer between the cover slip and the sample surface were varied.

\subsection{Experiments with agar phantoms}

Cavitation damage to tissue structures is usually assessed using ex-vivo or in-vivo tissue. However, this prohibits direct observation of damage in real time. Additionally,
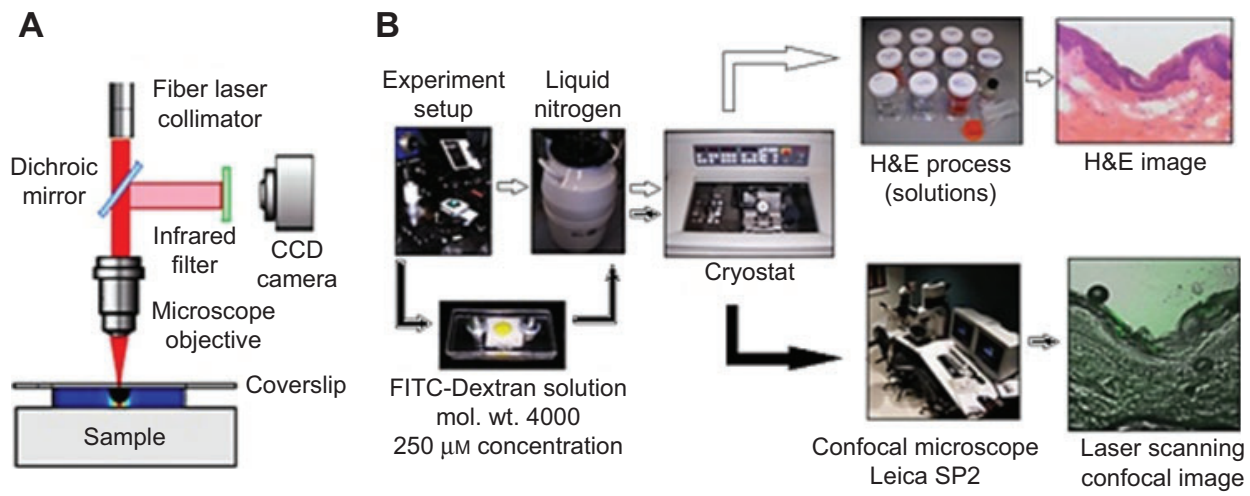

Figure 1 (A) Experimental setup to generate damage on agar gel and porcine skin. (B) Process to study the cavitation-induced damage on porcine skin. White arrows: path for obtaining sample histology (H\&E staining), black arrows: path for obtaining confocal fluorescent images. 
actual tissue requires preparation of histology slides to accurately identify lesions caused by cavitation, which is time-consuming and costly. Agar phantoms, on the other hand, are optically-transparent gels, allowing real-time visualization of cavitation while displaying mechanical properties similar to those of human skin. Therefore, agar gels were first used as a simple model to assess the range of parameters (laser power and layer thickness) that would be most appropriate for the porcine skin experiments, but for this purpose, it was necessary to guarantee good matching between the mechanical properties (elastic modulus) of the agar and skin models. Due to the large variability that exists in the mechanical properties of ex-vivo tissue related to factors such as skin hydration, age, anatomic location, etc., and despite the values reported in the literature [28], it was decided to measure independently the stress-strain relationship of all our samples. Agar and skin samples were subjected to simple compression tests, where the strain induced was measured as a function of the compressing force. $10 \mathrm{~mm}$-thick agar samples with concentrations varying between $1 \%$ and $5 \%$ were made in $52 \mathrm{~mm}$ diameter polystyrene dishes. A teflon plate was machined to fit tightly on top of the dish and a material testing machine (Mod. 3369; Instron, USA) pressed down on the teflon plate, recording the stress (force) and strain (compression) of each sample. Subsequently, thermocavitation experiments were performed on gel samples. The damage induced by thermocavitation on the surface of these agar phantoms was observed from the top and side view using an optical microscope (Mod. LSM700; Carl Zeiss MicroImaging, Germany), and assessed as a change in transparency and mass loss. Following this procedure, it was possible to determine that laser power between 170 and $200 \mathrm{~mW}$ and thickness of the solution layer fixed at $100 \mu \mathrm{m}$ would be most appropriate to use for the porcine skin samples.

\subsection{Experiments with porcine skin}

Once the porcine skin was exposed to thermocavitation, and in order to study the cavitation-induced damage on the SC and the subsequent drug penetration, three techniques were used: (1) tape stripping [29], (2) hematoxylin and eosin (H\&E) staining [30] and (3) laser scanning confocal imaging.

\subsubsection{Tape stripping}

Tape stripping [29] is a method well suited for studying the penetration of topically applied substances into the uppermost layer of the skin. It consists of peeling off the SC, cell layer by cell layer, attaching a new "scotch tape" at a time on the tissue surface and repeating the procedure until there is no evidence of drug attachment to the sticky side of the tape. The procedure was repeated 10 times on the treated skin area. A $250 \mu \mathrm{M}$ FITC-dextran solution (molecular weight, 4000 Da; Sigma Aldrich), was used as a hydrophilic, fluorescent drug surrogate to assess drug diffusion into the tissue. It was applied topically on the skin damaged by thermocavitation for $2.5 \mathrm{~h}$ before the tape stripping. The amount of topically applied substance removed by each tape strip was observed and photographed with a fluorescent microscope (Mod. MZFLIII; Leica, Germany). In order to determine penetration profiles, each digital fluorescent image was post-processed using a MATLAB program (MathWorks, USA); background noise was removed and pixel fluorescence intensity was summed up, which yields the total fluorescence intensity of each tape strip.

\subsubsection{H\&E staining}

All the samples of porcine skin exposed to thermocavitation were frozen immediately after laser exposure by dipping them into $\mathrm{LN}_{2}$ for $5 \mathrm{~min}$. Later, the tested samples were cut using a motorized cryostat (Mod. HM500 OMV; Mikron Instruments, USA) to obtain $20 \mu \mathrm{m}$ thick slices (see white arrows in Figure 1B). Half of the skin slices went through a typical H\&E staining histological process [30] and then were observed using an optical microscope (Mod. LSM700; Carl Zeiss MicroImaging, Germany) to obtain an overview of the structure of the tissue, enabling differentiation between normal or mechanically disrupted structures due to thermocavitation. Other staining techniques are available for histopathologic assessment following photothermal damage, such as nitro-blue tetrazolium chloride [31, 32], but in this study H\&E staining was used as it is a standard technique that is readily available in our laboratory and which is appropriate for determining thermomechanical disruption of tissue.

\subsubsection{Laser scanning confocal images}

The other half of the samples (see black arrows in Figure 1B), were exposed to an FITC-dextran solution for $2.5 \mathrm{~h}$ after laser exposure and before the skin was snap-frozen in $\mathrm{LN}_{2}$. Later, skin slices were obtained in the same way using the cryostat and then scanned by a confocal laser scanning microscope (Mod. TCS SP II; Leica, Germany) using 488/532 nm wavelengths for excitation 
and detection, respectively, in order to observe the penetration depth of the fluorescent solution into the SC and epidermis. The enhancing effect on the SC permeation of FITC-dextran was determined by comparing the cumulative total fluorescence intensity between the skin damage by thermocavitation (from different solutions' layers) and the control sample.

\section{Results and discussion}

\subsection{Agar gels}

\subsubsection{Effect of concentration}

Figure $2 \mathrm{~A}$ shows holes generated on the agar gels surfaces exposed to the CW laser at $153 \mathrm{~mW}$ of power, for $10 \mathrm{~s}$ and for a solution layer of $\sim 300 \mu \mathrm{m}$ placed in between the surface and a cover slip in all cases (Figure 1A). Each image corresponds to agar gel concentrations varying consecutively from $1 \%$ to $6 \%$. Based on previous work [20], this exposure time and laser parameters correspond to $\sim 4 \times 10^{4}$ low amplitude cavitation events, each generating maximum pressure waves of $\sim 0.1 \mathrm{MPa}$. For the $1 \%$ and $2 \%$ concentrations, the diameter of the holes was almost the same $(\sim 1 \mathrm{~mm})$, suggesting that this could be the maximum diameter produced by the shock waves under the conditions described above. This was confirmed through a separate experiment shown in Figure 2B, where 2\% agar samples with a $100 \mu \mathrm{m}$-thick $\mathrm{CuNO}_{4}$ layer solution were irradiated with $193 \mathrm{~mW}$ laser power for up to $8 \mathrm{~s}$. The resulting hole diameters were normalized because the observed behavior was the same regardless of the agar concentration, laser power or solution layer thickness. When concentration increased, the diameter, as well as the quality ("roundness"), of the hole became smaller until it disappeared at $6 \%$ concentration because the magnitude of the shock wave cannot tear off the agar surface.

\subsubsection{Effect of solution layer thickness}

Figure 3 shows a set of 3 holes, which were generated on an agar of $5 \%$ concentration, but different solution layer thicknesses (300, 200 and $100 \mu \mathrm{m})$ using a constant 170 $\mathrm{mW}$ laser power. A 5\% agar concentration was employed because it showed a good contrast between practically no damage produced by the thickest layer $(300 \mu \mathrm{m})$ to significant damage produced by the thinnest layer $(100 \mu \mathrm{m})$. This is because the shock wave amplitude is greatly attenuated near its source, i.e., the amplitude decays inversely proportional to its distance from the origin/source [20]. While we noticed shades of gray around the irradiation spots,
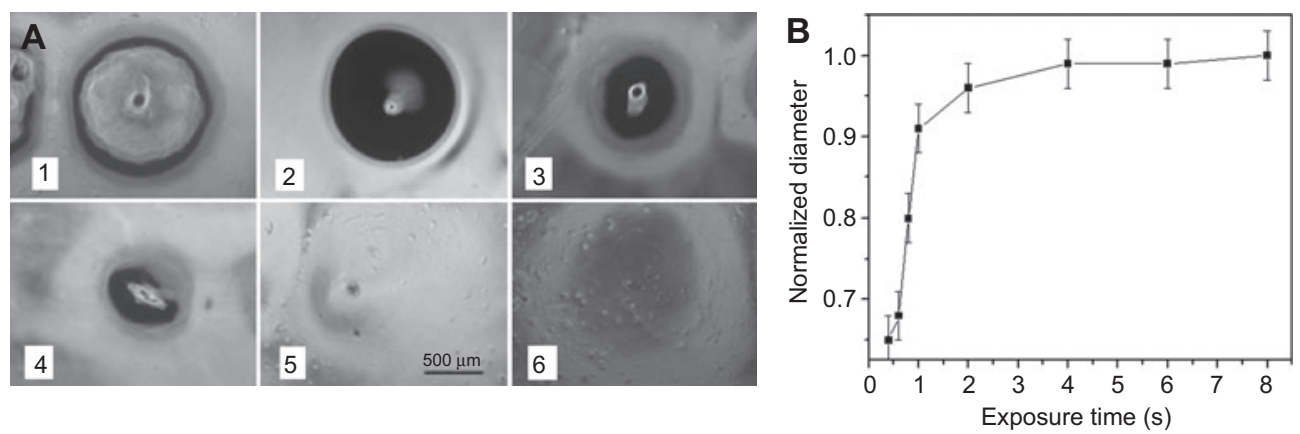

Figure 2 Damage generated on agar by the shockwave produced by thermocavitation. (A) Agar concentrations were: (1) $1 \%$, (2) $2 \%$, (3) $3 \%$, (4) $4 \%$, (5) $5 \%$ and (6) $6 \%$. The random spots on (5) and (6) are clusters of undissolved agar. (B) Normalized diameter of holes as a function of exposure time for a $2 \%$ agar concentration. The solution layer was $\sim 300 \mu \mathrm{m}$ and the laser power $153 \mathrm{~mW}$.
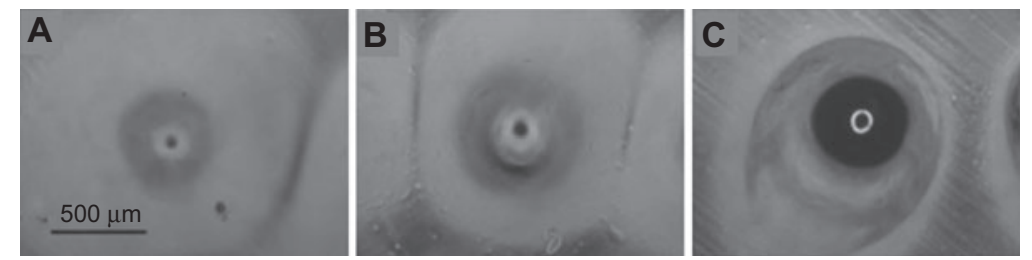

Figure 3 Holes on agar gel ( $5 \%$ concentration) at the same laser power (170 mW), but on different solution layers: (A) $300 \mu \mathrm{m}$, (B) $200 \mu \mathrm{m}$ and (C) $100 \mu \mathrm{m}$. 
possibly related to melting and re-gelling and/or entrapment of air bubbles, only visual inspection of diameter and depth of ablation were used as indicators of severe damage to the tissue models. Layer thickness smaller than $100 \mu \mathrm{m}$ were not explored because thermal damage risk increases as the radiation on the sample surface increases.

\subsubsection{Mechanical properties of agar versus porcine skin}

Figure 4 shows the stress-strain analysis for the respective agar concentrations. The elastic modulus $E$ of agar i.e., the slope of the stress-strain curves, decreases as the concentration of agar increases. The dashed lines indicate a linear fit to the elastic region. The values for $E$ were found to be 236, 90, 51, and $17 \mathrm{MPa}$ for the 1, 2, 3, and 5\% agar gel concentrations, respectively. For the particular case of $2 \%$ concentration, the stress-strain curve was consistent with the stress-strain curves of porcine skin reported in [33], where an elastic modulus of $\sim 95 \mathrm{MPa}$ was obtained, suggesting that this agar concentration possess similar mechanical properties to that of porcine skin. For this reason, most experiments were performed with this agar concentration.

\subsubsection{Effect of laser power}

The inset of Figure 5 shows an array of 5 holes generated on $2 \%$ concentration agar. Each column corresponds to different laser powers at the output of the microscope objective (see captions) and the exposure time was $10 \mathrm{~s}$ (see Figure 5). It is observed that for the lowest power, the diameter and depth of the holes were the largest $(\sim 1 \mathrm{~mm}$

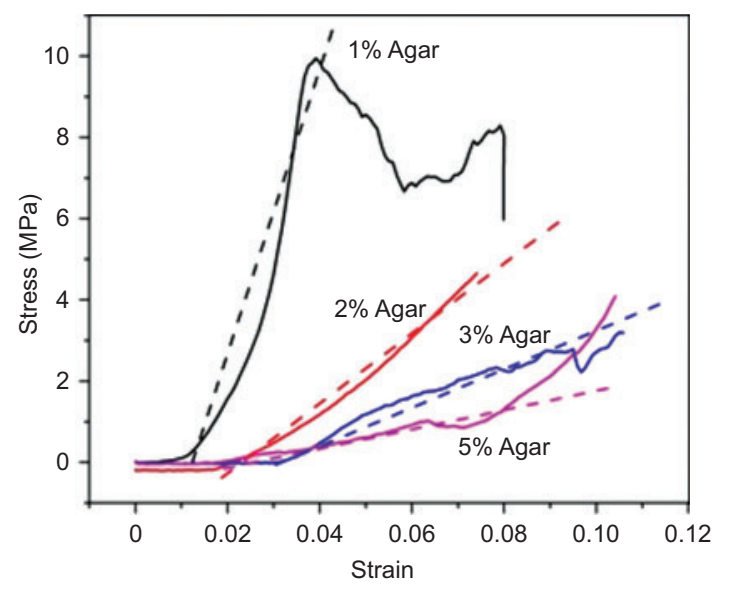

Figure 4 Stress-strain curves of several concentrations of agar gel. The dashed lines indicate an approximate linear fit within the elastic region of deformation.

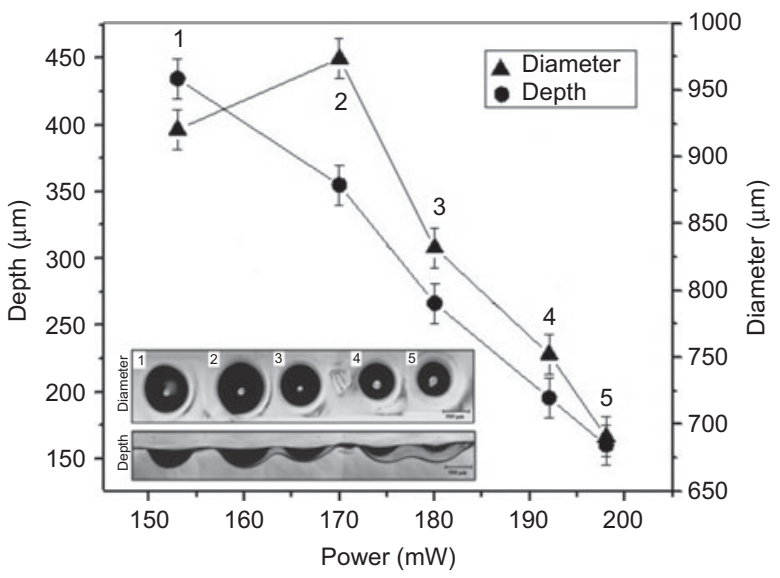

Figure 5 Depth and diameter of holes generated by thermocavitation in agar. Inset holes (top column) and depths (bottom column) generated on agar ( $2 \%$ concentration) by different laser powers: (1) $153 \mathrm{~mW}$, (2) $170 \mathrm{~mW}$, (3) $180 \mathrm{~mW}$, (4) $192 \mathrm{~mW}$ and (5) $198 \mathrm{~mW}$. The solution layer thickness was $300 \mu \mathrm{m}$.

diameter and $\sim 300 \mu \mathrm{m}$ depth) and, as power increased, both the diameter and depth decreased $(\sim 650 \mu$ m diameter and $\sim 150 \mu \mathrm{m}$ depth). This may seem contradictory at first sight, but in previous work it was shown that bubble's size (and therefore amplitude of the shock wave) increases as the power decreases because of the mismatch in the characteristic times of heat diffusion, $\tau_{\text {dif }}$, and cavitation time, $\tau_{c a v}$ [20]. When $\tau_{d i f}<\tau_{c a v}$ (low power), heat can be transferred to a larger volume, evaporating more water in the process and, therefore, a larger bubble is produced. Consequently, upon its collapse, a high amplitude shock wave is formed. In contrast, when $\tau_{d i f}$ is comparable or smaller than $\tau_{c a v}$ (high power), the vaporization volume is smaller because the bubble formation and growth ceases before heat can be diffused too far. Consequently, upon its collapse, the shock wave amplitude is also smaller. As expected, cavitation frequency increases with power because of the shorter time it takes for the bubbles to collapse and make the liquid available for a subsequent evaporation process [20]. Here, a congruent trend is observed: as laser power increases, the cavitation bubble size, diameter and depth decrease, suggesting that the formation of smaller bubbles' crater diameters and depths corresponds to shock waves of lower amplitude as shown in the inset.

\subsection{Porcine skin}

\subsubsection{Tape stripping}

Figure 6 shows the fluorescence intensity distribution of each tape strip, which is correlated to the penetration of 


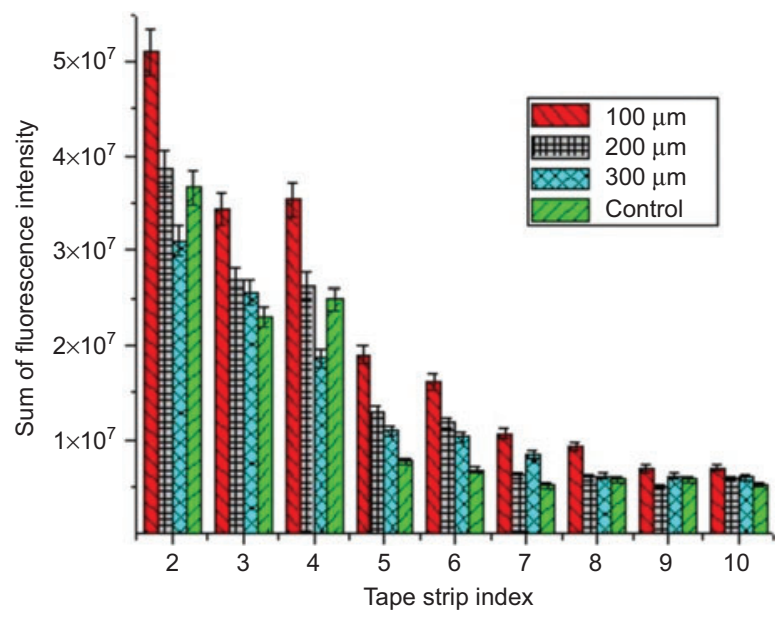

Figure 6 Penetration profiles of topically applied FITC-dextran on the stratum corneum. The porcine skin was exposed to $193 \mathrm{~mW}$ of CW laser radiation and different thickness of solution layer

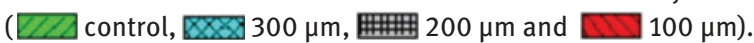

FITC-dextran solution into the skin. It should be noted that the first tape strip contained almost a complete cell layer of SC and it was ignored because its fluorescence is easily affected by highly variable skin surface conditions. The size of the error bars simply denotes the standard deviation of the intensity measurements of the samples measured (between 3 and 5 for each experiment). However, the true uncertainty and repeatability of the tests is much larger, given the inherent randomness of the tests. Estimating a maximum significance level of 30\%, a $t$-test of two independent populations comparing the control to the $100 \mu \mathrm{m}$ layer experiments reveals a $p$-value of 0.26 , indicating that these two populations were statistically different. Despite the variability of this method and its partially qualitative nature, this experiment suggests that the penetration of FITC-dextran into the skin is greater for thermocavitation induced with the thinnest layer of solution $(100 \mu \mathrm{m})$ particularly for the upper layers, congruent with the cavitation-induced damage on the SC results obtained with the agar.

\subsubsection{H\&E and confocal fluorescent imaging: control}

Figure 7 shows the images of the histological structure of the tissue by the H\&E technique (top), and the confocal fluorescent microscope to observe the penetration of the FITC-dextran solution topically applied on the porcine skin surface (bottom) for $2.5 \mathrm{~h}$. Each image was taken from adjacent slices of porcine skin samples (20 $\mu$ m thick) not exposed to thermocavitation (control). The H\&E image shows intact SC and epidermis, and the confocal fluorescent image shows the solution's fluorescence (FITC-dextran) is confined to the surface of the SC, showing that SC is the principal barrier to drug permeation into the skin.

\subsubsection{H\&E and confocal fluorescent imaging: thermocavitation}

The experiments described above were repeated, and this time the samples were inspected after they were exposed to thermocavitation, using again a $\sim 100 \mu \mathrm{m}$ thick solution topically applied on the skin surface for $2.5 \mathrm{~h}$. To observe the damage more readily, the laser beam was displaced along the surface to generate lines on the surface of the SC (Figure 8) - as opposed to the holes created on the agar gels by keeping the laser beam fixed. Then, by taking cross sectional cuts, the damage generated by thermocavitation can be observed. The lines were performed by moving the skin sample at a speed of $0.5 \mathrm{~mm} / \mathrm{s}$ using the maximum power available from the laser system (193 $\mathrm{mW}$ ). This ensures continuous lines over the SC because the cavitation's frequency at this power is the largest possible, albeit smaller damage, as shown clearly in the agar models (Figure 5). Subsequently, two adjacent slices (20 $\mu \mathrm{m}$ thick) were obtained from the same sample; the first went through the process of H\&E staining and the second was analyzed in the confocal microscope to observe the penetration of the fluorescent solution into the SC (Figures 9 and 10).

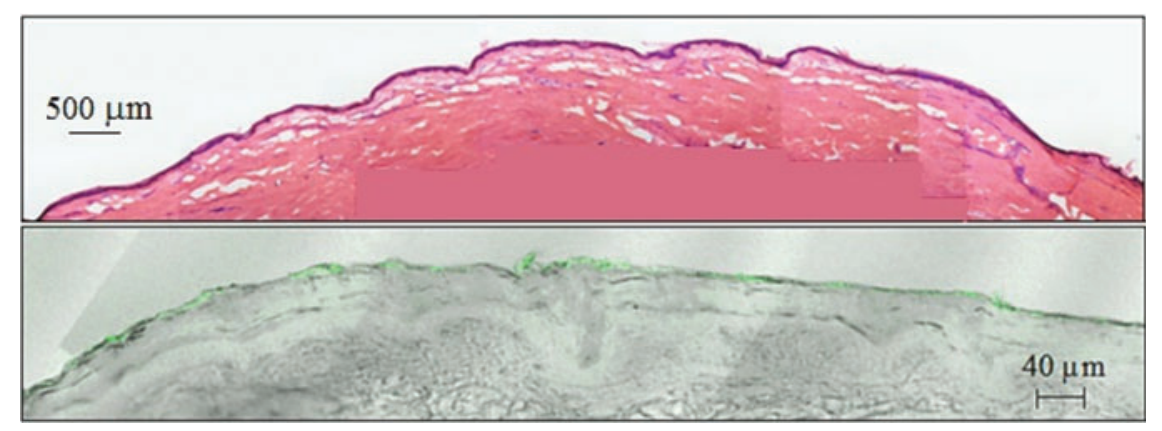

Figure 7 Porcine skin not exposed to thermocavitation. H\&E control (top) and confocal microscope fluorescent image (bottom). 


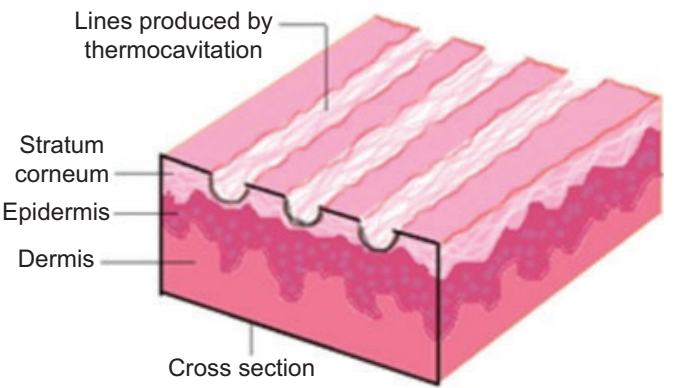

Figure 8 Lines produced on the stratum corneum surface by thermocavitation. Later the sample is cut transversally using the motorized cryostat to get $20 \mu \mathrm{m}$ thick slices.

Figure 9 is a complete view of an H\&E image of a selected tissue slice, where it is possible to observe the damage on the SC in at least three locations marked by 1 , 2 and 3, respectively. This damage is assessed, first, by the evident peel off of the uppermost skin layers (SC and epidermis) and then further verified under the optical microscope. Figure 10A-C shows a close up of these three locations (top) and their corresponding (adjacent) confocal fluorescent images (bottom). Location 1 in Figure 9 shows that the damage generated along this channel is almost superficial (H\&E image) and, consequently, the solution penetration depth is minimal (see Figure 10A, fluorescent image). However, in the contiguous lines (locations 2 and 3 ) the damage increases. There is an abrupt tearing of the SC of $\sim 80-100 \mu \mathrm{m}$ in diameter, and possibly even minimal damage to the upper epidermis. This damage allows the FITC-dextran solution to penetrate deeper $(-40-60 \mu \mathrm{m})$ as shown in the fluorescent images of Figures 10B and 10C, respectively.

\section{Discussion}

The discrepancy between regions 1 and 2-3 (Figure 9) may seem contradictory because the experiment was performed under the same conditions, so damage of the same size was expected in all three cases. The explanation for this behavior is that the maximum bubble radius and, consequently, the shock wave amplitude generated at the moment of collapse depends largely on variations in the sample thickness, flatness, and even a slight tilt of the sample from its ideal perpendicular position with respect to the laser beam. These variables affect the

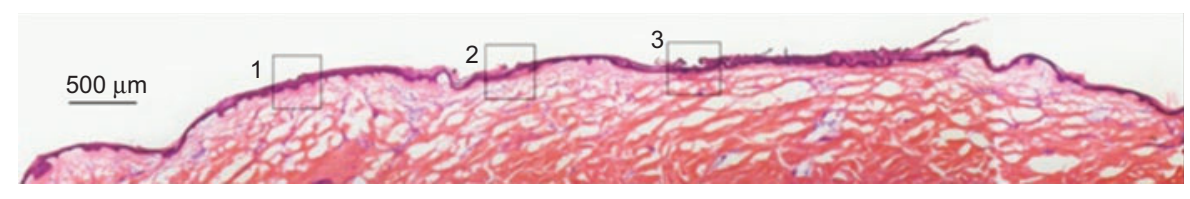

Figure 9 Histological structure of porcine skin exposed to thermocavitation. The solution layer was $100 \mu \mathrm{m}$ and the laser power $193 \mathrm{~mW}$.
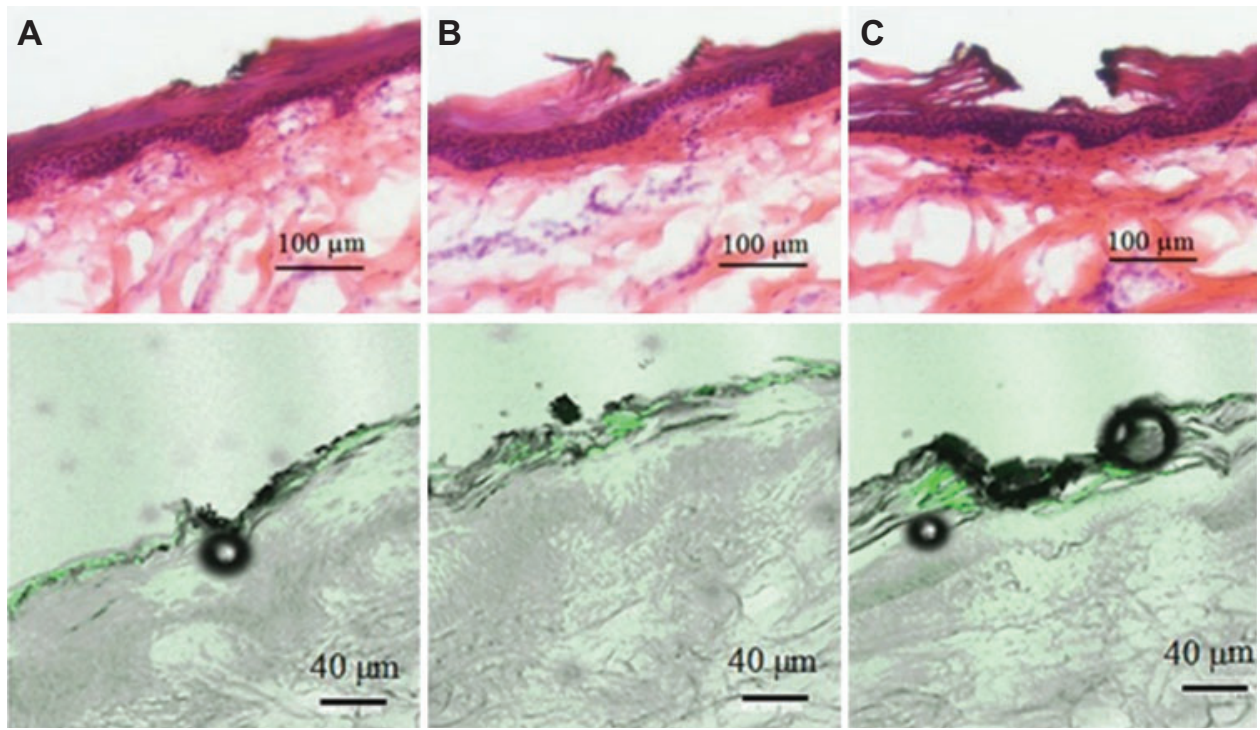

Figure 10 Close up of Figure 9. H\&E images (top) and laser scanning confocal images for skin sections of $20 \mu \mathrm{m}$ (below), where the FITCdextran solution was topically applied for $2.5 \mathrm{~h}$. 
bubble size and therefore shock wave amplitude, producing different damage scenarios for each channel. Also, the maximum damage obtained on the SC $(\sim 80-100 \mu \mathrm{m}$ diameter) is much smaller than that obtained in the agar, possibly due to subtle differences on mechanical properties between both materials but, also, due to the mismatch in the number of cavitation events between the two types of samples used. A modest increase in the number of cavitations events and/or a reduction of the laser power is anticipated to increase the depth of the holes.

An issue of concern was the dark region noticeable in the H\&E and fluorescent images near the periphery of the ruptured tissue. It was speculated that such damage could be induced by excessive heat transfer from the solution layer and/or laser absorption itself which could have ultimately burned the skin surface. Furthermore, since the SC has lower water content than epidermis, it was plausible that ablative damage could be induced to the $\mathrm{SC}$ at lower temperatures and shorter exposure times than those needed to induce damage to the epidermis. To investigate this hypothesis, two subsequent analyses were performed using a finite element heat transfer model (COMSOL Multiphysics, V 4.2; COMSOL, USA). For this purpose, human skin thermophysical properties reported in [34] were used and water properties were assumed for the $\mathrm{CuNO}_{4}$ solution. The first study was aimed at computing the theoretical maximum temperature that could be reached within the $100 \mu \mathrm{m}$ thick solution layer in between the cover slip and skin surface during laser exposure of a $193 \mathrm{~mW}$ CW laser. Once the temperature across the layer was known, the second study was aimed at determining the theoretical cumulative thermal damage to the tissue underneath using an Arrhenius tissue damage model [34].

Figure $11 \mathrm{~A}$ shows the thermal map within the $110 \mu \mathrm{m}$ thick solution layer at the end of $240 \mu \mathrm{s}$. This time corresponds to the cavitation time, i.e., the time since the laser is turned on until cavitation occurs [20]. Figure 11B shows the temperature distribution at the skin surface (i.e., the SC) and illustrates that the maximum temperature reached is $\sim 72^{\circ} \mathrm{C}$ in $\sim 240 \mu \mathrm{s}$, congruent with the cavitation time obtained experimentally [20]. Thereafter, the temperature decreases as the bubble expands and collapses, and the cycle repeats again at a frequency of $\sim 4 \mathrm{kHz}$ for these conditions. Upon bubble collapse and subsequent convective mixing, it is expected that this temperature decreases even at a higher rate, but this cannot be computed with this model. Nevertheless, our measurements indicate that temperature increase of the solution after a few minutes of illumination reaches about $5^{\circ} \mathrm{C}$, and the current study suggests that thermal damage is unlikely to occur within the epidermis given that a temperature as high as $72^{\circ} \mathrm{C}$ is reached but only for a few fractions of millisecond.

In order to assess the thermal damage on the skin, the Arrhenius damage integral $(\Omega)$ was then computed, represented by Eqn. 1:

$\Omega(t i)=\ln \left(\frac{C(0)}{C(t i)}\right)=A \int_{0}^{t i} \exp \left[-\frac{E_{a}}{R T(\mathrm{x}, \mathrm{z}, \mathrm{t})}\right] d t$

where $\Omega(t i)$ is the thermal damage parameter at $t=t i ; C(0)$ is the number of undamaged cells at time $0 ; C(t i)$ is the number of undamaged cells at time $t ;$; $A$ is the frequency factor or the molecule collision rate; $E_{a}$ is the activation
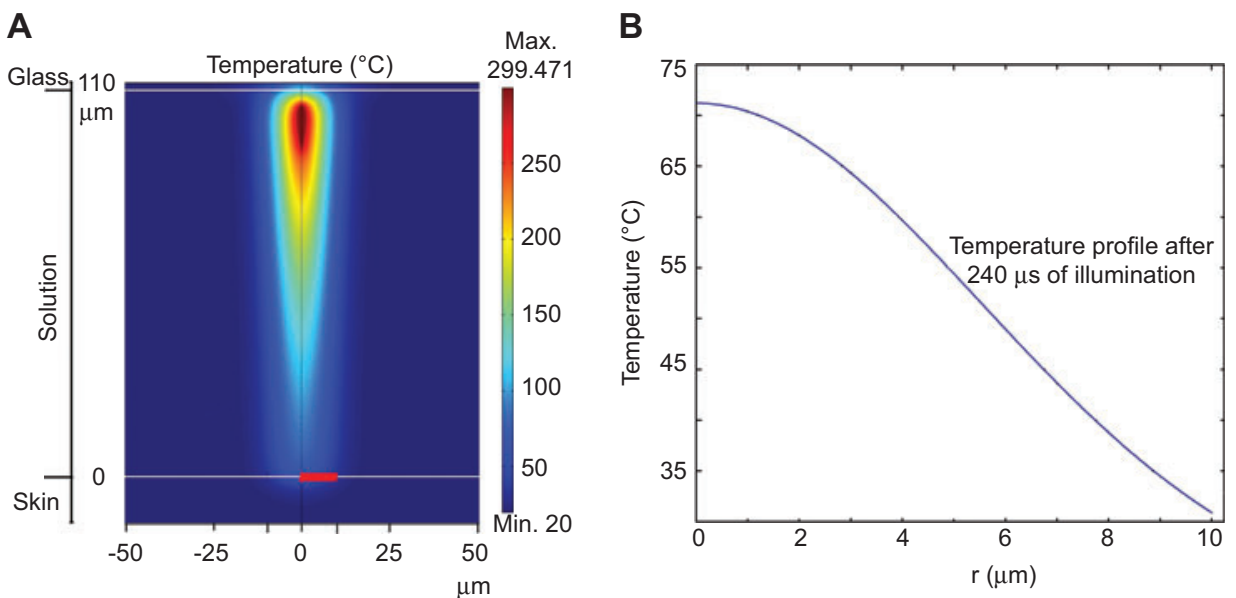

Figure 11 (A) Temperature profile within a $110 \mu \mathrm{m}$ thick $\mathrm{CuNO}_{4}$ solution and (B) at the skin surface (stratum corneum) at the end of $240 \mu \mathrm{s}$ pulse of a $193 \mathrm{~mW}$ laser. The simulation ends when the temperature of $300^{\circ} \mathrm{C}$ is reached and thermocavitation occurs ( $\left.240 \mu \mathrm{s}\right)$. The radial temperature profile on the skin's surface (red bar) shows a rapid decrease from the $72^{\circ} \mathrm{C}$ at the beam centre to almost room temperature within $10 \mu \mathrm{m}$. 


\begin{tabular}{llllc} 
Specimen & & $E_{a}(\mathrm{~J} / \mathbf{m o l} / \mathrm{K})$ & $A(\mathbf{1} / \mathbf{s})$ & $\boldsymbol{\Omega}(t i=\mathbf{2 0 0} \mathbf{m s})$ \\
\hline Porcine skin & Back & 525,500 & $2.126 \mathrm{E}+81$ & 1.14203 \\
& Belly & 393,500 & $1.151 \mathrm{E}+61$ & 0.75226 \\
& Ear & 586,700 & $5.240 \mathrm{E}+91$ & 13.88818 \\
& Face & 471,000 & $4.575 \mathrm{E}+72$ & 0.47972 \\
Human blood & Flank & 401,200 & $1.501 \mathrm{E}+61$ & 0.06598 \\
& & 455,000 & $7.680 \mathrm{E}+66$ & 0.00021 \\
\hline
\end{tabular}

Table 1 Mean thermal damage $(\Omega)$ induced to porcine skin after $200 \mathrm{~ms}$ of $\mathrm{CW}$ laser exposure to a constant temperature of $72^{\circ} \mathrm{C}$. Laser wavelength $=975 \mathrm{~nm}$; power $=193 \mathrm{~mW}$. $E_{a}$ and $A$ values obtained from [35].

energy in $\mathrm{J} / \mathrm{mol}$; and $R$ is the universal gas constant of $8.3144 \mathrm{~J} / \mathrm{mol} / \mathrm{K}$.

From visual inspection of the histology samples, a region of damage of $\sim 100 \mu \mathrm{m}$ in diameter was observed (Figure 10C), which means that $\sim 800$ cavitation events took place during approximately $200 \mathrm{~ms}$. Using this time and the maximum temperature computed before $\left(72^{\circ} \mathrm{C}\right)$, the $\Omega$ values shown in Table 1 were computed. These values correspond to the mean thermal damage calculated over a region of $10 \mu \mathrm{m}$ in radius at the interface skinsolution (see the horizontal red line in Figure 11A) for skin harvested from different anatomic locations.

Note that only values of $\Omega \geq 1$ correspond to cell damage $>63 \%$ relative to the existing undamaged cells before exposure, and lesser values are normally considered inconsequential or correspondent to reversible damage at best. Two values $>1$ were computed: for ear and back skin. Although there is always some uncertainty as to the anatomic location of the skin samples used, back and ear skin is easily recognizable, and the samples used for this study were not. Therefore, this study also suggested that the damage observed in the samples is unlikely related to heated solution and/or laser-induced epidermal burning. Finally, burning by light absorption is also unlikely since the beam diameter at the skin $(\sim 20 \mu \mathrm{m})$ is much smaller than the damaged region $(\sim 100 \mu \mathrm{m})$. So the reason for the darker region in Figure 10 remains to be determined, but laser burning or thermal damage by a hot liquid is ruled out.

\section{References}

[1] Chaussy C, Brendel W, Schmiedt E. Extracorporeally induced destruction of kidney stones by shock waves. Lancet 2(8207):1265-8.

[2] Coleman AJ, Saunders JE, Crum LA, Dyson M. Acoustic cavitation generated by an extracorporeal shockwave lithotripter. Ultrasound Med Biol 1987;13(2):69-76.

\section{Conclusions}

The damage observed on agar and porcine skin appears to be congruent with the relationship between laser power, focal point, cavitation frequency and extent of damage observed in previous studies $[19,20]$ and, therefore, related to the emission of shock waves produced at the collapse of the thermocavitation bubbles. In particular, the shockwave amplitude produced by thermocavitation decreases with laser power, but the width and depth of the damaged zone increases.

In this study, the greatest damage induced to the agar phantoms is produced with the lowest laser power $(\sim 153$ $\mathrm{mW})$ and thinnest solution layer $(-100 \mu \mathrm{m})$ used.

Similar laser and solution layer settings led to porcine skin damage of $\sim 80-100 \mu \mathrm{m}$ in diameter, which was sufficiently large to break the $\mathrm{SC}$ and allow the penetration of $4 \mathrm{kDa}$, FITC-dextran to depths of $\sim 40-60 \mu \mathrm{m}$. However, the extent of damage induced on porcine (and presumably human) skin is likely dependent on variations in the sample thickness, flatness, and even a slight tilt of the sample from its ideal perpendicular position with respect to the laser beam. A systematic study to assess this issue is warranted.

Some uncertainty remains in relation to the dark region noticeable in the H\&E and fluorescent images near the periphery of the ruptured tissue. First-approach numerical simulations suggest that those regions cannot be caused by the temperatures and exposure times corresponding to laser absorption within the liquid solution and subsequent superheating, thus laser burning or thermal damage by a hot liquid is ruled out.

From an application point of view, this combination of CW laser and absorbing solution is in fact quite convenient because it would reduce the cost relative to other more sophisticated TDD methods.

Acknowledgements: The authors acknowledge financial support from CONACyT-Mexico under project (2010156876 JCRSJ).

Received May 18, 2012; revised June 18, 2012; accepted June 19, 2012

[3] Yong DZ, Lipkin ME, Simmons WN, Sankin G, Albala DM, Zhong P, Preminger GM. Optimization of treatment strategy used during shockwave lithotripsy to maximize stone fragmentation efficiency. J Endourol 2011;25(9):1507-11.

[4] Alexandrov AV. Ultrasound-enhanced thrombolysis for stroke: clinical significance. Eur J Ultrasound 2002;16(1-2):131-40. 
[5] Góes JC, Landecker A. Ultrasound-assisted lipoplasty (UAL) in breast surgery. Aesthetic Plast Surg 2002;26(1):1-9.

[6] Speed CA. Therapeutic ultrasound in soft tissue lesions. Rheumatology (Oxford). 2001;40(12):1331-6.

[7] Hadjiargyrou M, McLeod K, Ryaby JP, Rubin C. Enhancement of fracture healing by low intensity ultrasound. Clin Orthop Relat Res 1998;355 (Suppl):S216-29.

[8] Guzmán HR, Nguyen DX, Khan S, Prausnitz MR. Ultrasoundmediated disruption of cell membranes. I. Quantification of molecular uptake and cell viability. J Acoust Soc Am 2001;110(1):588-96.

[9] Miller DL, Quddus J. Sonoporation of monolayer cells by diagnostic ultrasound activation of contrast-agent gas bodies. Ultrasound Med Biol 2000;26(4):661-7.

[10] Sundaram J, Mellein BR, Mitragotri S. An experimental and theoretical analysis of ultrasound-induced permeabilization of cell membranes. Biophys J 2003;84(5):3087-101.

[11] Wu J, Ross JP, Chiu JF. Reparable sonoporation generated by microstreaming. J Acoust Soc Am 2002;111(3):1460-4.

[12] Shen N, Schaffer CB, Datta D, Mazur E. Photodisruption in biological tissues and single cells using femtosecond laser pulses. In: IEEE, editor. Conference on Laser and ElectroOptics: CLEO 2001 (IEEE Conference Proceedings). Baltimore: I.E.E.E. Press; 2001, p. 403-4.

[13] Vogel A, Busch S. Time-resolved measurements of shock wave emission and cavitation-bubble generation in intraocular laser surgery with ps- and ns-pulses. In: Blake JR, BoultonStone JM, Thomas NH, editors. Bubble dynamics and interface phenomena: Proceedings IUTAM Symposium, held at Birmingham, UK, 6-9 September 1993 (Fluid mechanics and its applications). Amsterdam: Kluwer Academic Publishers; 1994, p. 105-17.

[14] Ueda H, Mutoh M, Seki T, Kobayashi D, Morimoto Y. Acoustic cavitation as an enhancing mechanism of low-frequency sonophoresis for transdermal drug delivery. Biol Pharm Bull 2009;32(5):916-20.

[15] Tezel A, Mitragotri S. Interactions of inertial cavitation bubbles with stratum corneum lipid bilayers during low-frequency sonophoresis. Biophys J 2003;85(6):3502-12.

[16] Tang H, Wang CC, Blankschtein D, Langer R. An investigation of the role of cavitation in low-frequency ultrasound-mediated transdermal drug transport. Pharm Res 2002;19(8): 1160-9.

[17] Sun F, Anderson R, Aguilar G. Stratum corneum permeation and percutaneous drug delivery of hydrophilic molecules enhanced by cryopneumatic and photopneumatic technologies. J Drugs Dermatol 2010;9(12):1528-30.

[18] Rastopov SF, Sukhodolsky AT. Cluster nucleation in the process of $\mathrm{cw}$ laser-induced thermocavitation. Phys Lett A 1990;149(4):229-32.

[19] Rastopov SF, Sukhodolsky AT. Sound generation by thermocavitation-induced cw laser in solutions. Proc SPIE 1990;1440:127-34.

[20] Ramirez-San-Juan JC, Rodriguez-Aboytes E, Martinez-Canton AE, Baldovino-Pantaleon 0, Robledo-Martinez A, Korneev N, Ramos-Garcia R. Time-resolved analysis of cavitation induced by cw lasers in absorbing liquids. Opt Express 2010;18(9):8735-42.

[21] Korneev N, Rodriguez Montero P, Ramos-García R, RamirezSan-Juan JC, Padilla-Martinez JP. Ultrasound induced by cw laser cavitation bubbles. J Phys: Conf Ser 2011;278:012029. doi: 10.1088/1742-6596/278/1/012029.

[22] Ramirez-San-Juan JC, Padilla-Martinez JP, Zaca-Moran P, Ramos-Garcia R. Micro-hole drilling in thin films with Cw low power lasers. Opt Mater Express 2011;1(4): 598-604.

[23] Yoon J, Park D, Son T, Seo J, Nelson JS, Jung B. A physical method to enhance transdermal delivery of a tissue optical clearing agent: combination of microneedling and sonophoresis. Lasers Surg Med 2010;42(5):412-7.

[24] Padilla-Martinez JP, Aguilar G, Ramirez-San-Juan JC, Ramos-García R. Temporal evolution of thermocavitation bubbles using high speed video camera. Proc SPIE 2011;8097: 809727.

[25] Sacchi CA. Laser-induced electric breakdown in water. JOSA B 1991;8(2):337-45.

[26] Barnes PA, Rieckhoff KE. Laser induced underwater sparks. Apll Phys Lett 1968;13:282-4.

[27] Byun KT, Kwak HY, Karng SW. Bubble evolution and radiation mechanism for laser-induced collapsing bubble in water. Jpn J Appl Phys 2004;43(9A):6364.

[28] Subhash G, Liu Q, Moore DF, Ifju PG, Haile MA. Concentration dependence of tensile behavior in agarose gel using digital image correlation. Exp Mech 2011;51(2): 255-62.

[29] Lademann J, Jacobi U, Surber C, Weigmann HJ, Fluhr JW. The tape stripping procedure - evaluation of some critical parameters. Eur J Pharm Biopharm 2009;72(2): 317-23.

[30] Carson FL, Hladik C. Histotechnology: a self-instructional text. 3rd edition. Chicago: ASCP Press; 2009.

[31] Khan MH, Sink RK, Manstein D, Eimerl D, Anderson RR. Intradermally focused infrared laser pulses: thermal effects at defined tissue depths. Lasers Surg Med 2005;36(4): 270-80.

[32] Neumann RA, Knobler RM, Pieczkowski F, Gebhart W, Enzyme histochemical analysis of cell viability after argon laserinduced coagulation necrosis of the skin. J Am Acad Dermatol 1991;25:991-8.

[33] Uzer G, Ho A, Clark RAF, Fu-pen C. Mechanical properties of pig skin. Proceedings of the SEM Annual Conference June 1-4, 2009 Albuquerque, New Mexico, USA. <http:// sem-proceedings.com/09s/sem.org-SEM-2009-Ann-Confs078p03-Mechanical-Properties-Pig-Skin.pdf $>$.

[34] Shafirstein G, Bäumler W, Lapidoth M, Ferguson S, North $\mathrm{PE}$, Waner M. A new mathematical approach to the diffusion approximation theory for selective photothermolysis modeling and its implication in laser treatment of port-wine stains. Lasers Surg Med 2004;34(4):335-47.

[35] Xu F, Seffen KA, Lu TJ. Temperature-Dependent mechanical behaviors of skin tissue. IJCS 2008;35:1-10. <http://www. iaeng.org/IJCS/issues_v35/issue_1/IJCS_35_1_13.pdf $>$. 